\title{
El Ciclo de Indagación: una metodología para la investigación ecológica aplicada y básica en los sitios de estudios socio-ecológicos a largo plazo, y más allá
}

\author{
The Inquiry Cycle: a research methodology for applied and basic ecology at sites of Chilean \\ long-term socio-ecological studies, and beyond
}

\author{
Peter Feinsinger ${ }^{\mathrm{a}, \mathrm{b} *}$ \\ *Autor de correspondencia: Pasaje El Astillero No 8, (4401) Vaqueros, Salta, Argentina, peter.feinsinger@nau.edu \\ ${ }^{a}$ Northern Arizona University, Department of Biological Sciences, Flagstaff, Arizona EE.UU. \\ ${ }^{\mathrm{b}}$ Wildlife Conservation Society, Latin American and Caribbean Program, Bronx, New York, EE.UU.
}

\begin{abstract}
SUMMARY
The Inquiry Cycle represents an integrated methodology for field investigations under continuous development in Latin America since 1994. The investigator first formulates the research question following an explicit sequence of observation, conceptual framework and site-specific concern. The research question must comply with five criteria that directly frame the design of the field study in itself and later the discussion of results (reflection). In particular, the question specifies the design factors and the response units or experimental units, and the response variables to be recorded in each response unit. These elements lead to a detailed, 17-step process of study design. Similarly, the reflection phase follows an ordered, cautious, and creative review of the results once obtained, examines all possible causal factors, and cautiously but creatively suggests possible extrapolations to other contexts and scales. All steps of the Inquiry Cycle as a research methodology also serve as the template for writing a complete and objective thesis or publication. For research involving forest management, wildlife management, agroecology, conservation biology, or another aspect of applied field biology, the Inquiry Cycle adds a fourth explicit step (application). It is now the Applied Inquiry Cycle, codifying the process of adaptive management. Today the two Cycles provide the Chilean Long-term Socio-ecological Research Network with a research protocol for applied as well as basic studies.
\end{abstract}

Key words: adaptive management, Applied Inquiry Cycle, design of field research, Inquiry Cycle, research methodology.

\section{RESUMEN}

El Ciclo de Indagación es una metodología de investigación integrada que ha ido desarrollándose en América Latina desde 1994. El Ciclo comienza por una Pregunta de trabajo, formulada mediante una secuencia explícita de tres pasos previos: el planteo de la Observación, el Concepto de Fondo y la Inquietud Particular. La redacción de la Pregunta cumple con cinco criterios que condicionan el diseño de la investigación (Acción) y la discusión de los hallazgos (Reflexión). La Pregunta debe precisar tanto los factores de diseño como las unidades de respuesta o experimentales, además de las variables de respuesta que se registrarán. Esto conduce a un proceso exigente de 17 pasos del diseño del estudio. De manera análoga, la Reflexión sigue una secuencia explícita que consiste en revisar los hallazgos, proponer causas posibles de estos y sugerir cautelosa y creativamente, posibles extrapolaciones a otros contextos y ámbitos. Todos los elementos del Ciclo de Indagación como metodología explícita de investigación sirven también para la redacción completa de una tesis o publicación. Si la investigación trata sobre el manejo forestal, la conservación biológica u otra disciplina de la biología de campo aplicada, al Ciclo de Indagación se le agrega otro paso, la Aplicación. De esta forma se convierte en el Ciclo de Indagación Aplicada, que guía el desarrollo de investigaciones con fines de manejo adaptativo. En la Red Chilena de Estudios SocioEcológicos a Largo Plazo, el Ciclo de Indagación ofrece hoy un protocolo para investigaciones tanto básicas como aplicadas.

Palabras clave: ciclo de indagación, ciclo de indagación aplicada, diseño de investigación, manejo adaptativo, metodología de investigación.

\section{INTRODUCCIÓN}

Para los investigadores y estudiantes que trabajan en el Parque Nacional Fray Jorge, la Estación Biológica Senda Darwin en la Isla Grande de Chiloé, el Parque Etnobotánico Omora en la Isla Navarino y otros sitios de estudios ecológicos a largo plazo en Chile -o en otros paisajes de
América Latina- una de las principales inquietudes que surge es: ¿cómo concebir una investigación científica de alta calidad y a su vez de alta utilidad para la ecología aplicada y/o la básica? Una inquietud constante de muchos profesores que están contribuyendo a la formación de jóvenes investigadores a través de América Latina es también: ¿cómo enseñar a nuestros alumnos a diseñar y desarrollar 
su investigación y analizar, interpretar y aplicar los resultados de forma cautelosa y creativa (Ford 2000)? Durante las dos décadas en que trabajé en mis propias investigaciones y la formación de estudiantes de postgrado en universidades norteamericanas asumí, junto a gran parte de mis colegas, que la combinación del método científico hipotético deductivo (véase por ejemplo, Fretwell 1972, Underwood 1990, Quinn y Keough 2002), del diseño experimental bien planteado y de la bioestadística aplicada fielmente, proveería de una herramienta de investigación rigurosa y apta para cualquier tesis de postgrado o proyecto científico.

En 1992 un colega y este autor enfrentaron el reto de plantear un curso novedoso dirigido a biólogos ecuatorianos que trabajaban en temas relacionados con la conservación biológica, un curso que tratara explícitamente del diseño de estudios no experimentales (estudios de observación, ver Shadish et al. 2002) en paisajes remotos. Para construir dicho curso, hubo que reevaluar la confianza que previamente habían depositado en las aproximaciones tradicionales de la investigación ecológica tal como se enseñaba en la mayoría de las universidades. Esta reevaluación de la metodología de investigación en estudios de campo, iniciada en ese primer curso (febrero de 1993), ha continuado hasta el presente, a través de decenas de cursos impartidos a alumnos de pregrado y postgrado, docentes universitarios, otros profesionales y personal de diversas áreas protegidas de América latina.

En los primeros de estos cursos, la base epistemológica para la capacitación de ecólogos básicos y aplicados, y para sus investigaciones, consistió en el método hipotético deductivo y técnicas afines (Feinsinger 2001). Simultáneamente, se trabajaba con docentes de escuelas primarias en la Enseñanza de Ecología en el Patio de la Escuela (EEPE), para quienes se propuso una metodología de investigación más sencilla: el Ciclo de Indagación (Feinsinger et al. 1997). El Ciclo de Indagación desde su inicio consistió en tres pasos: la formulación de una Pregunta de trabajo, la Acción de tomar los datos que responden a dicha Pregunta y la Reflexión sobre los resultados y sus posibles implicaciones (figura 1). Con el transcurso del tiempo se dio cuenta de que el Ciclo de Indagación también puede ser útil para llevar a cabo investigaciones científicas profesionales (Feinsinger 2004). Así, el Ciclo de Indagación fue perfeccionado de manera rigurosa como metodología de investigación, incorporando su uso cada vez más en los cursos a nivel de postgrado y profesional. Ya por el año 2005, por razones discutidas en otros trabajos (Feinsinger 2013, Feinsinger y Ventosa Rodríguez 2014) el Ciclo de Indagación y el Ciclo de Indagación Aplicada, su variante para el manejo y la conservación, habían suplantado las demás metodologías en los cursos avanzados sobre el diseño de estudios de campo, además de ser fundamental en la capacitación en investigación científica de otros públicos, tales como guardaparques, docentes, escolares, campesinos e indígenas (Arango et al. 2009, Feinsinger 2010ab).

En este ensayo se espera mostrar que la aparente sencillez del Ciclo de Indagación no debilita su rigor científico respecto a otras metodologías de investigación académicas (véase Feinsinger 2013), sino que lo fortalece. Actualmente, es la metodología usada en numerosos estudios de campo aplicados y básicos, realizados por profesionales, alumnos de pregrado y postgrado en universidades de al menos ocho países desde Cuba al extremo sur de Chile. Sin embargo, en algunos ámbitos académicos el desconocimiento del ciclo como se presentó hace casi una década (Feinsinger 2004), y de su desarrollo posterior, ha lleva-

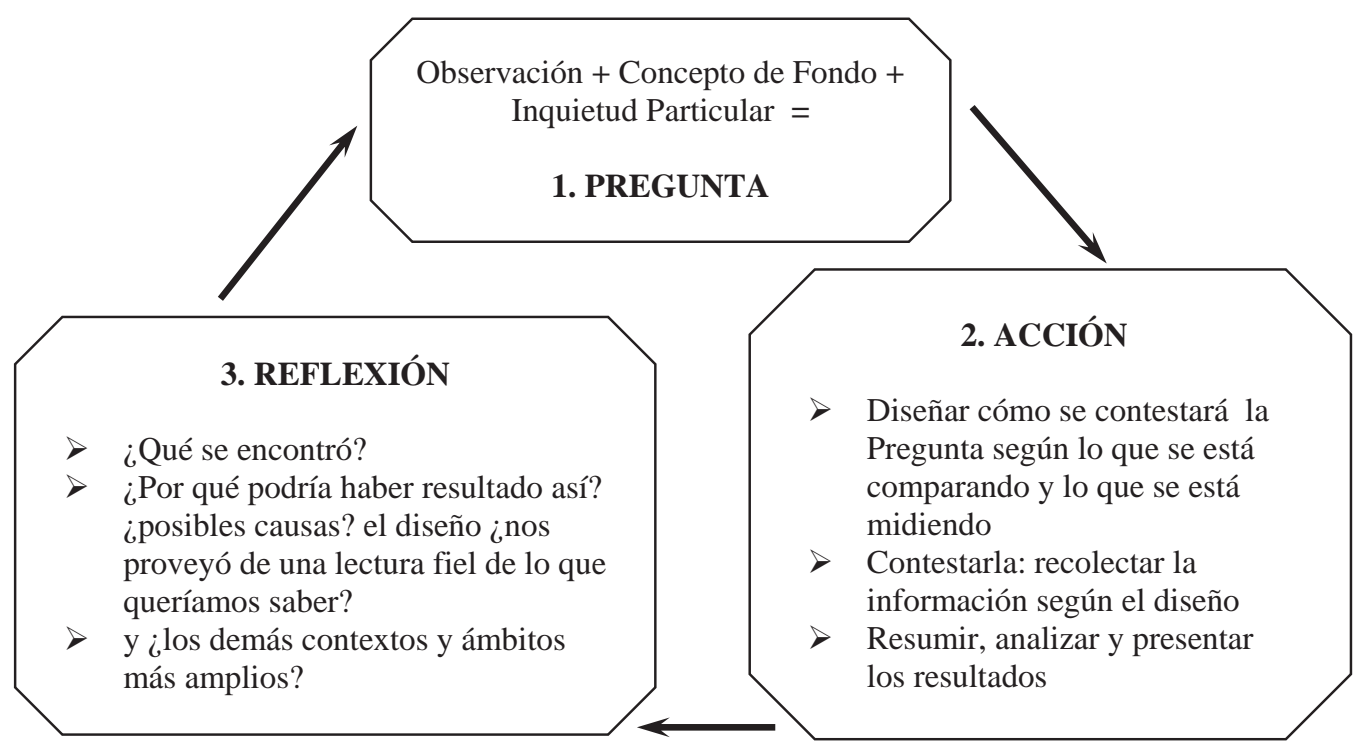

Figura 1. El Ciclo de Indagación (modificado ligeramente de Feinsinger 2004, 2012).

The Inquiry Cycle (slightly modified from Feinsinger 2004, 2012). 
do a interpretaciones erróneas (véase Marone y Galetto 2011). Con este resumen de los puntos claves del Ciclo de Indagación y Ciclo de Indagación Aplicada espero aportar elementos a los ecólogos aplicados y básicos para valorar con imparcialidad esta metodología de investigación y evaluar sus posibles ventajas para plantear, realizar, interpretar y redactar investigaciones de campo.

\section{LA PREGUNTA DE TRABAJO}

Cómo llegar a la Pregunta. La Pregunta de trabajo (figura 1) es el inicio y el cimiento del Ciclo de Indagación (Feinsinger 2004). Tiene su origen en tres procesos mentales explícitos.

Al recorrer su entorno, el ecólogo nota algún fenómeno que le llama la atención y despierta su interés o inquietud. Lo redacta explícitamente como la Observación, la que puede considerar más de un solo elemento del entorno.

La Observación se relaciona con el Concepto de Fondo (figura 1) o marco teórico, conocimiento o noción que ya está archivada en la mente del ecólogo, parte de su formación académica, su conocimiento de la literatura científica y/o su experiencia previa. El Concepto de Fondo debe ser una afirmación global, no restringida a un lugar, tiempo o especie particular. En algunos casos, es bastante análoga a un paradigma o teoría. En otros casos es similar a la hipótesis científica $\left(H_{c}\right)$ del método hipotético deductivo de Popper (Feinsinger 2013) y puede emplear el mismo lenguaje u otro lenguaje formal del marco teórico de la ecología u otro campo, tal como la "hipótesis de la perturbación intermedia" (Connell 1978). Sin embargo, a diferencia de la $H_{c}$, el Concepto de Fondo admite excepciones, dados los múltiples factores y contingencias que caracterizan los fenómenos ecológicos en el campo (Quinn y Dunham 1983, Lawton 1999). Por ende, suele incluir la frase: por lo general (...). Si la Observación incluye más de un elemento del entorno, el Concepto de Fondo también trata sobre más de un solo fenómeno.

La Inquietud Particular (figura 1) se refiere al ámbito cercano al investigador, llevando la propuesta global (el Concepto de Fondo) a una que no lo es tanto y que hace referencia a la Observación original. Así como el Concepto de Fondo, la Inquietud Particular no tiene que redactarse según reglas estrictas. Puede escribirse como una interrogante acerca de las posibles causas o consecuencias de lo observado, por ejemplo: ¿cómo incide (incidía, incidió, incidiría, afecta, afectaba, afectó, afectaría) (...)?, ¿será que (...)?, ¿¿cuáles factores inciden (o, incidían) en (...)?, ¿por qué $(\ldots)$ ? o ¿̇a qué se debe $(\ldots)$ ?, entre muchas otras posibilidades. Puede presentarse como una propuesta, por ejemplo: es posible que lo observado refleje el efecto de (...). Sin embargo, es imprescindible recalcar que la Inquietud Particular no es la Pregunta de trabajo (ver la siguiente subsección). La Inquietud Particular casi nunca se puede responder directamente por medio de una investigación. De hecho, una misma Inquietud Particular puede conducir a numerosas indagaciones distintas. El autor y sus colegas han encontrado que uno de los problemas epistemológicos, metodológicos y biológicos más graves y más comunes en las investigaciones en ecología básica y aplicada, es confundir una Inquietud Particular con la Pregunta de trabajo (o, con la predicción del método hipotético deductivo), e intentar diseñar y realizar un estudio para responder directamente a la Inquietud Particular, especialmente una que contenga las palabras ¿̇or qué (...)?. Esto no sólo dificulta la toma de datos, sino también el análisis, interpretación y reflexión (ver abajo).

La secuencia explícita de formulación y presentación de cada uno de los tres elementos que acompañan a la Pregunta de trabajo (Observación, Concepto de Fondo e Inquietud Particular) debe seguirse rigurosamente.

La redacción de la Pregunta en sí. A diferencia de los tres elementos que la acompañan, la Pregunta de trabajo debe redactarse con sumo cuidado según cinco reglas explícitas. ¿Por qué? Porque las palabras precisas de la Pregunta determinan directamente el diseño del estudio, sea este un experimento o un estudio de observación (ver abajo), y fijarán el ámbito de la discusión, interpretación y aplicación de los resultados. Cuatro de las cinco reglas para formular la Pregunta ya han sido ampliamente discutidas (por ejemplo, Arango et al. 2009, Feinsinger 2004, Feinsinger et al. 2010ab), por lo que no hace falta discutirlas aquí en detalle. La quinta, vigente desde enero 2014 (Feinsinger y Ventosa Rodríguez 2014), no requiere explicaciones mayores. Brevemente, la Pregunta de trabajo debe ser: 1) contestable directamente mediante la toma de nuevos registros en el campo; 2) comparativa; 3) interesante o atractiva en dos sentidos, que no tenga una respuesta conocida y que no exija trabajo excesivo o abrumador en relación a su importancia y 4) redactada con un lenguaje sencillo. Finalmente, debe ser 5) coherente: sus elementos claves (lo que se comparará y se medirá) deben derivarse claramente de la cadena de razonamiento desde la Observación y el Concepto de Fondo hasta la Inquietud Particular.

En su segunda regla, la Pregunta debe precisar lo que se comparará, los diferentes niveles del factor de diseño (es decir, la variable de ingreso o, en experimentos, el factor de tratamiento). Debe precisar la naturaleza del sujeto unitario e independiente de lo que se comparará: la unidad de respuesta o, en un experimento, la unidad experimental (Hurlbert 2009, Feinsinger y Ventosa Rodríguez 2014). Además, debe precisar lo que se observará (o "medirá") dentro de la unidad de respuesta, que no es más que la variable de respuesta o variable de egreso, cumpliendo así con la primera regla. En el análisis estadístico, el factor de diseño será la variable independiente y la variable de respuesta será la variable dependiente. Según la Observación, Concepto de Fondo e Inquietud Particular pueden existir dos o más factores de diseño.

La precisión de las palabras es esencial, ya que lo que se está comparando y lo que se está midiendo condicionan 
el diseño del estudio de campo, el análisis estadístico de los datos, la inferencia estadística y, finalmente, la interpretación y aplicación de los resultados (Feinsinger y Ventosa Rodríguez 2014). La manera más eficiente y precisa de redactar la Pregunta, sea sobre un estudio de observación o sobre un experimento (Shadish et al. 2002), suele ser: ¿cómo varía $Y$ (la variable de respuesta) entre las unidades de respuesta $i$ en relación con los diferentes niveles de $X$ (el factor de diseño)? (Feinsinger 2012, 2013).

También es imprescindible que el ecólogo tenga clara la diferencia fundamental entre lo que se comparará y lo que se medirá, en particular, la diferencia entre la unidad de respuesta (que se refiere a lo que se está comparando) y la unidad de evaluación (donde se toma el registro de la variable de respuesta), porque puede haber más de una unidad de evaluación por una misma unidad de respuesta, es decir, submuestras. El ecólogo que no separa de manera clara los dos conceptos a partir de la Pregunta, corre el riesgo de caer en el frecuente error de la seudorreplicación (Hurlbert 2009).

\section{Ejemplo sencillo de la secuencia completa.}

- Observación: el quebracho colorado, Schinopsis lorentzii (Griseb.) Engl., es una especie de alto valor forestal que habita en el Chaco semiárido del noroeste de la Argentina. Casi todas sus poblaciones en las diferentes zonas de bosque chaqueño tienen una historia de tala selectiva o indiscriminada, pero todavía presentan adultos y renovales. Hay ganado vacuno y caprino presente en muchas de las zonas de bosque pero no en todas.

- Concepto de Fondo: por lo general el ganado incide negativamente en la estructura y dinámica de especies de árboles, debido a su consumo de renovales o el simple pisoteo de ellos. Por otro lado, el ganado puede incrementar la dispersión de propágulos y/o estimular el rebrote de raíces de algunas especies.

- Inquietud Particular: ¿cómo incidirá el ganado vacuno/ caprino en el futuro de las poblaciones remanentes del quebracho colorado en el Chaco semiárido de Argentina?

- Pregunta de trabajo: durante la época húmeda de 2015, en el Chaco semiárido de las provincias de Salta y Santiago de Estero, Argentina ¿cómo varían la densidad (número por $100 \mathrm{~m}^{2}$ ) de plántulas y juveniles de quebracho colorado, entre zonas de bosque con presencia de ganado vacuno/caprino y zonas sin ganado, pero con historias semejantes de tala ?

Según esta Pregunta, la unidad de respuesta es una zona de bosque de Chaco semiárido, con o sin ganado. La palabra "zonas" indica que habrá réplicas de cada uno de los dos niveles del factor de diseño entremezclados entre sí, es decir, muchas zonas grandes de bosque sin ganado y muchas zonas con ganado. Por contraste, la unidad de evaluación no será la zona de bosque como un todo, sino el área fija determinada por un cuadrante, parcela rectangular o par- cela circular, donde el investigador realizará el conteo de plántulas y juveniles de quebracho colorado. Es casi seguro que el ecólogo submuestreará, empleando varias unidades de evaluación para conseguir datos representativos de una misma unidad de respuesta (la zona de bosque) como un todo. Ya que la Pregunta define la unidad de respuesta, será difícil (aunque no imposible) confundirla con la unidad de evaluación y caer en la trampa de la seudorreplicación.

Relación con el producto final. La secuencia desde Observación hasta Pregunta, sin que se empleen esas etiquetas explícitas, es el bosquejo completo de la Introducción bien redactada del proyecto de investigación y luego la tesis o publicación que derive del estudio. Simplemente, hay que darle cuerpo agregando citas bibliográficas que apoyen el Concepto de Fondo y otras que corroboren la observación.

\section{LA ACCIÓN}

El diseño. La Acción que sigue a la Pregunta (figura 1) consiste en tres etapas: el diseño del estudio que mejor conteste la Pregunta, la toma de datos según el diseño y el tratamiento de los datos. El proceso de diseño consiste en 17 pasos secuenciales, sea el diseño de una indagación de campo aplicada o de una básica. Dichos pasos se detallan en otro trabajo (Feinsinger y Ventosa Rodríguez 2014). Sin embargo, de manera resumida, los pasos pueden ser agrupados en cuatro fases.

En la primera fase (de tres pasos), el investigador considera el ámbito espacio-temporal del estudio y ajusta la Pregunta según las realidades de la historia natural y las escalas de espacio y tiempo que están a su alcance. Decide entre realizar un estudio de tiempo fijo o uno de monitoreo, es decir, de tiempo indefinido. Además, debe seleccionar entre un experimento controlado y un estudio de observación (Shadish et al. 2002). Un experimento controlado debe seguir los protocolos rigurosos del llamado diseño experimental (por ejemplo, Meade 1988, Quinn y Keough 2002). Por contraste, el diseño de un estudio de observación (uno no experimental) no puede seguir esos protocolos y no puede, obviamente, llamarse diseño experimental.

La segunda fase del proceso de diseño (de cinco pasos) trata exclusivamente de la definición precisa de lo que se quiere comparar: el factor de diseño, sus niveles y la unidad de respuesta, la selección de las unidades de respuesta en el espacio y el tiempo y los ajustes a la Pregunta según tales definiciones.

Una vez definida y diagramada la comparación, el ecólogo pasa a los cuatro pasos de la tercera fase: lo que se registrará ("medirá") al llegar a una unidad de respuesta determinada. La preocupación que debe tener en mente durante estos pasos es, ¿estoy midiendo lo que quiero medir? Es muy probable que la respuesta sea no, en particular si la investigación trata de animales. Así es imprescindible revisar consciente y cuidadosamente los pasos y elecciones de qué registrar, con cuál metodología y en qué unidad de evaluación. 
Una manera de evitar equivocaciones es la realización de un premuestreo, uno de los cinco pasos finales para afinar el diseño. Otros pasos de esta cuarta fase buscan definir el tamaño de la muestra, la ética de muestreo, la selección a priori del tipo de análisis descriptivo y la presentación gráfica de los datos, además de la forma de realizar la inferencia estadística.

La toma de datos y refinamiento de la Pregunta. Finalmente, el ecólogo realiza la toma cuidadosa de los datos, siguiendo el mejor diseño para responder a su Pregunta (figura 1). Durante el transcurso del estudio puede ser que unos elementos cambien, por efecto de problemas no anticipados, tanto de la logística como de la historia natural. El ecólogo debe considerar las limitaciones que estas realidades imponen sobre el diseño original y reevaluar o ajustar la forma de la Pregunta para reflejar las posibilidades de la toma de datos. Puede ser necesario reducir el ámbito espacial y/o temporal del estudio. Sin embargo, un diseño bien pensado, aun con los ajustes necesarios, permite un estudio sólido y riguroso.

Análisis, resumen y presentación de datos. Una vez tomados los datos (figura 1), es necesario volver a pensar en la lógica y el significado biológico del análisis estadístico, la inferencia estadística y la manera de presentar los gráficos y cuadros que resumen los resultados. Estos pasos, resumidos en Feinsinger (2012), se discuten en detalle en el texto de Feinsinger y Ventosa Rodríguez (2014).

Relación con el producto final. Los elementos claves del proceso de diseño deben estar explícitos en la redacción de las secciones de métodos y resultados de la tesis o publicación. Naturalmente, no hay que detallar cada uno de los pasos. Sin embargo, es necesario explicitar todos los elementos que se compararon y dibujar un esquema de la ubicación de las unidades de respuesta de los diferentes niveles del factor de diseño. Es necesario justificar la elección de la variable de respuesta, la metodología usada para obtenerla, la naturaleza de la unidad de evaluación y la del submuestreo si se ha realizado. Es imprescindible diferenciar bien entre la unidad de respuesta (el sujeto que comparó) y la de evaluación (donde se tomó la medición). En muchas tesis y trabajos publicados es imposible descifrar cuál es cuál mientras que en otras ocasiones es evidente que se confundieron, cayendo en el error de la seudorreplicación (ver Ramage et al. 2013).

\section{LA REFLEXIÓN}

El puente: los resultados aquí y allá. La Reflexión (figura 1) es un paso desafiante, cualquiera que sea la metodología de investigación, ya que por un lado debe ser creativa e innovadora y por otro debe ser realizada con precaución, diferenciando explícitamente entre lo que es (los datos en sí, sean representativos o no de la población de datos de que provienen -lo que es a menudo incognoscible) y lo que podría ser (los posibles fenómenos explicativos y extrapolaciones a otros ámbitos, Feinsinger 2012). Efectivamente, la Reflexión en el Ciclo de Indagación debe comenzar volviendo a visitar explícitamente lo que es, es decir, realizando un resumen retrospectivo de los resultados. Se inicia con la pregunta, ¿cuáles fueron las tendencias más llamativas (o falta de ellas) de los resultados? y ¿cuáles fueron las excepciones más llamativas a dichas tendencias? Esto sirve como un puente entre la presentación de los resultados en detalle (la última etapa de la Acción) y las especulaciones que constituirán la mayor parte de la Reflexión (ver más adelante). También aquí se suele comparar los principales resultados con los de trabajos semejantes ya publicados, en particular aquellos realizados en ambientes semejantes.

¿Por qué podría haber resultado así? Los posibles fenómenos explicativos. En esta fase, el ecólogo empieza a cuestionar los resultados. Considera todas las posibles explicaciones de cómo ellos podrían haber resultado así, citando trabajos teóricos y empíricos según corresponda. Primeramente, vuelve a la cadena de razonamiento que condujo inicialmente a la Pregunta de trabajo:

1. ¿El fenómeno que causó las tendencias podría estar relacionado con el Concepto de Fondo (y en muchos casos con la Inquietud Particular también)?

Tanto si los resultados parecen reflejar o no la influencia del factor relacionado con el Concepto de Fondo, el ecólogo debe preguntarse:

2. ¿Los fenómenos que causaron las tendencias (o falta de ellas) entre los resultados podrían haber sido otros, es decir, factores que no están relacionados con el Concepto de Fondo (ni con la Inquietud Particular)? ¿Cuáles pudieron haber sido dichos fenómenos y cómo pudieron haber ocasionado las tendencias observadas o la carencia de ellas? Farji-Brener (2009) recalca la importancia de esta etapa de la reflexión.

A menos que los resultados sean muy consistentes, sin ninguna excepción a las tendencias generales, el ecólogo puede especular sobre aquellos datos atípicos:

3. Los datos que se apartan de las tendencias generales ¿pudieron haber sido ocasionados por sucesos, condiciones u otros fenómenos puntuales y particulares a una sola o unas pocas unidades de respuesta? ¿Cuáles pudieron haber sido dichos fenómenos particulares y cómo pudieron haber afectado las unidades de respuesta en cuestión? Estas reflexiones sobre los datos excepcionales pueden ser tanto o más interesantes que las tendencias generales, por lo que no deben despreciarse, subestimarse o (peor aún) botarse (Farji-Brener 2009).

Finalmente, el ecólogo debe considerar explícitamente la posibilidad de que los resultados reflejen artefactos del 
diseño del estudio en vez de la biología, a pesar del cuidado con que realizó el diseño:

4. Las tendencias de los resultados y/o las excepciones llamativas ¿pudieron haber resultado de efectos ocultos de un diseño inadecuado o mal hecho? Es decir, ¿será posible que el diseño, incluyendo la selección de unidades de respuesta y la metodología de medición, otorgara una lectura inapropiada de lo que se quiere saber para responder la Pregunta? Si fuera así ¿cómo sería un diseño mejor? Esta instancia tiene un fuerte componente de modestia, ética y autocrítica (ver FarjiBrener 2009).

Cabe volver a recalcar la diferencia fundamental entre un estudio de observación y un estudio experimental (Shadish et al. 2002). El Ciclo de Indagación puede aplicarse a ambos, pero hay una diferencia notoria en la Reflexión. Si existe un diseño experimental riguroso y una selección cuidadosa de las unidades experimentales, el ecólogo que realiza un experimento bien controlado no entra en una discusión de las fases 1 y 2 (Feinsinger 2013). Las únicas posibles causas de las tendencias observadas en los resultados son los tratamientos experimentales, derivados directamente del Concepto de Fondo. Sin embargo, todavía se debe reflexionar sobre las posibles causas de datos atípicos (fase 3) y sobre el significado biológico de la variable de respuesta (fase 4). Si los resultados de un experimento bien controlado muestran tendencias con significado biológico, es un claro indicio de que el fenómeno definido por el Concepto de Fondo (y a menudo la Inquietud Particular) tiene significado biólogo -dentro de las condiciones precisas del experimento, las que pueden representar bien o mal la realidad. Por otro lado, la carencia de tendencias indica que el fenómeno carece de significado biológico -dentro de las condiciones experimentales.

Sin embargo, muchas indagaciones en ecología son estudios de observación. Esto significa que aún cuando los resultados muestren las tendencias que se esperarían si el fenómeno considerado en el Concepto de Fondo (y la Inquietud Particular) fuera responsable de esa tendencia (fase 1 de este paso de Reflexión), el ecólogo no puede afirmar que la causa fue el fenómeno considerado por su Concepto de Fondo (e Inquietud Particular). Es decir, no debe caer en la falacia de afirmar el consecuente, ya que otros fenómenos reales e imposibles de controlar (fase 2 de este paso) podrían haber producido las tendencias observadas. Por ejemplo, si el ecólogo de bosques encuentra una diferencia marcada de la densidad de plántulas y/o juveniles de quebracho colorado entre zonas de bosque chaqueño con y sin ganado, no puede concluir que la causa es el ganado. Es posible que diferencias preexistentes en la estructura de la vegetación den cuenta de la diferencia en la densidad y estructura de población del quebracho colorado entre los bosques. La redacción debe señalar abiertamente esta incertidumbre, especulando, por ejemplo, que es probable que las acciones del ganado contribuyan a las diferencias observadas entre las densidades de plántulas y juveniles en el estudio; sin embargo, también es posible que las diferencias resulten de (...).

De manera análoga, la carencia de tendencias en un estudio de observación no indica que el fenómeno asociado al Concepto de Fondo no tenga significado biológico en el sistema investigado, porque otros fenómenos imposibles de controlar podrían haber contrarrestado sus efectos durante la indagación (fase 2 de este paso de reflexión). Por ende, es imprescindible que en un estudio de campo de observación se consideren cuidadosa y creativamente, todos los elementos ( 1 a 4 ) que cuestionan ¿por qué podría haber resultado así? Además, las fases 2 - 4, en particular, constituyen una fuente de ideas innovadoras, cada una de las cuales es capaz de estimular indagaciones novedosas y avances significativos en la ecología u otro campo. Asimismo, cabe destacar que la carencia de tendencias llamativas, sea que provenga de un experimento o un estudio de observación, debe también ser informada y publicada (Loehle 1987, Farji-Brener 2009).

¿Qué podría suceder más allá del estudio en sí? Habiendo extraído el jugo de los resultados en cuanto a los factores que podrían haberlos causado, ahora el ecólogo debe aprovechar los resultados y las nuevas ideas generadas como un trampolín para especular sobre lo que podría pasar, bajo condiciones diferentes a las de la indagación en sí. Si la investigación fue experimental, primero debe preguntarse: ¿qué podrían tener que ver los resultados, con lo que sucede cotidianamente en este mismo ámbito? ya que siempre es posible que los factores controlados durante el experimento tengan más importancia en la vida real que el tratamiento experimental, a pesar de la elegancia y rigurosidad interna del experimento.

Tanto si la indagación es experimental o de observación, esta fase es la más creativa de la reflexión. Aquí el investigador se pregunta cómo podrían ser los resultados si se realizara un estudio semejante a escalas espaciales mayores (o menores) que la de la indagación en sí, en otros tiempos (horarios, temporadas, años con otro clima), bajo otras condiciones físicas (días con más o menos lluvia, otros suelos, otras pendientes, otras exposiciones, entre muchas otras), bajo otras condiciones biológicas (diferentes abundancias, diferentes variedades de seres vivos vecinos, con o sin depredadores o parásitos, más mutualistas o menos, entre muchas otras), en otros hábitats (formaciones vegetales o latitudes, o hábitats con más o menos perturbación antrópica) y sobre seres vivos diferentes a los estudiados y con diferentes historias de vida (y comportamientos), entre muchos otros temas. En cualquiera de estos casos, es necesario tener bien presente que se trata de un salto desde lo encontrado bajo las condiciones de la indagación cumplida hacia lo que se podría encontrar bajo otras condiciones. No se puede plantear afirmaciones sobre los otros entornos o sobre un ámbito más amplio. No se puede afirmar que las diferencias encontradas en la den- 
sidad de plántulas y juveniles de quebracho colorado entre bosques con diferentes historias de ganadería, demuestran que el quebracho colorado del Chaco semiárido -o peor, que la vegetación de todos los bosques secos tropicalesestá amenazado por la actividad ganadera.

¿Podría haber aplicaciones? Aunque un estudio basado en el Ciclo de Indagación representa la ciencia básica, sin fines explícitos de conservación o manejo, a menudo la Reflexión incluye extrapolaciones imaginativas y realistas (pero no afirmaciones) acerca de posibles aplicaciones de los resultados, por ejemplo en los contextos de suelos, cultivos, animales domésticos, áreas protegidas, otros espacios verdes, la ecología urbana o temas particulares a la conservación biológica, por ejemplo, el manejo de bosques, el manejo de fauna o el diseño de zonas de amortiguación en un área protegida.

Propuestas de nuevas líneas de investigación y nuevas Preguntas. La Reflexión no debería concluir solo con especulaciones. Como lo que indica la última flecha del Ciclo de Indagación (figura 1), las especulaciones y extrapolaciones explícitas llevan a una lluvia de nuevas ideas de investigación. Como mínimo estimulan numerosas Inquietudes Particulares, algunas a partir del primer Concepto de Fondo y otras referidas a Conceptos de Fondo alternativos. Idealmente, el ecólogo concluye su Reflexión proponiendo nuevas Preguntas de trabajo que ya cumplen con las cinco reglas. Otro trabajo (Feinsinger y Ventosa Rodríguez 2014) presenta un ejemplo de Reflexión que concluye con la propuesta de nuevas Preguntas de trabajo.

Relación con el producto final. La discusión bien redactada de una tesis o trabajo publicado es nada más y nada menos que la Reflexión, enriquecida por la integración objetiva y ética de la bibliografía teórica y empírica. Una discusión siempre debería comenzar por un párrafo de puente, resumiendo y recalcando lo que es (Feinsinger 2012). De ahí en adelante debe mostrar un cambio en el uso de los verbos, o el uso del condicional (podría ser, podría haber sido) o de construcciones que reflejan la incertidumbre (es probable, es posible, se propone que), e idealmente sigue la consideración de todas las fuentes posibles de causalidad de los resultados y todas las extrapolaciones posibles de lo encontrado. Finalmente, las discusiones de los artículos más renombrados en ecología concluyen con propuestas innovadoras de líneas de investigación, derivadas del desarrollo de los párrafos anteriores.

\section{EL CICLO DE INDAGACIÓN APLICADA}

Propósito. Si la investigación tiene el fin explícito de analizar amenazas a la conservación, evaluar pautas de manejo alternativas o brindar información importante para la toma de decisiones sobre el manejo de bosques, la conservación biológica u otro campo relacionado con el entorno local (por ejemplo, la agroecología), se agrega otro paso al Ciclo de Indagación. El resultado es el Ciclo de Indagación Aplicada (figura 2), discutido ampliamente por Feinsinger et al. (2010ab) y Feinsinger y Ventosa Rodríguez (2014).

Las consignas del Ciclo de Indagación (figura 1), es decir, la secuencia de Observación, Concepto de Fondo, Inquietud Particular y Pregunta que considere las cinco pautas, el diseño, la toma y análisis de datos y la mayor parte de la Reflexión, son también parte del Ciclo de Indagación Aplicada. Las diferencias entre los dos ciclos se encuentran hacia el final de la Reflexión, donde se enfatiza la meta explícita del Ciclo de Indagación Aplicada: influir en la toma de decisión (figura 2), implementar la pauta de manejo elegida y monitorear el resultado. Aquí los puntos de partida de las indagaciones futuras no surgen directamente de la Reflexión sino de los resultados del monitoreo, una vez implementada la Aplicación, en particular de los resultados inesperados. A menudo el cuarto paso consiste en una nueva indagación completa, donde lo que se compara es lugares (u otros contextos) en que se han implementado la pauta y contextos de control (Feinsinger y Ventosa Rodríguez 2014). Muchas de las indagaciones bajo el Ciclo de Indagación Aplicada, aunque no todas, tratan sobre el manejo adaptativo (Holling 1978).

\section{Ejemplo de la secuencia completa.}

- Observación: en el Parque Provincial Moconá (Misiones, Argentina) la recuperación de la vegetación en la pista de aviación abandonada es muy lenta.

- Concepto de Fondo: las aves frugívoras y otros frugívoros pueden incidir en el proceso de la recuperación de un claro creado por la perturbación del bosque, al defecar las semillas de especies del bosque primario. Por lo general, las aves defecan posadas sobre perchas (ramas). Por tanto, tienden a depositar menos semillas en el centro del claro que en el borde, donde se pueden posar sobre las ramas de los árboles que se encuentran allí. Así, la recuperación de la vegetación suele tardar más en el centro. Sin embargo, la provisión de perchas artificiales para las aves puede acelerar la llegada de semillas al centro y por ende su recuperación.

- Inquietud Particular: ¿La provisión de perchas artificiales en la pista de aviación abandonada del Parque Provincial Moconá podría facilitar la recuperación de la vegetación?

- Pregunta de trabajo: en la pista de aviación abandonada del Parque Provincial Moconá ¿cuáles y cuántos renovales de especies arbóreas se encuentran en parcelas a diferentes distancias del borde norte de la pista, con perchas artificiales y sin perchas, durante el lapso de cuatro años (2008-2012)?

Se puede notar que esta indagación, que fue propuesta por guardaparques y condujo a un excelente diseño rigurosamente pensado, aborda un experimento realista y relevante. Pone a prueba una posible pauta de manejo (la 
provisión de perchas artificiales), la que se implementará y será monitoreada por los mismos investigadores, si esa es su decisión luego de reflexionar sobre los resultados.

\section{¿LA SENCILLEZ Y LA RIGUROSIDAD CIENTÍFICA SON INCOMPATIBLES?}

En resumen, los Ciclos de Indagación y de Indagación Aplicada pueden conducir a investigaciones completas, sólidas y rigurosas, aunque la demostración convincente requeriría argumentos adicionales (Feinsinger 2013, Feinsinger y Ventosa Rodríguez 2014) y, sobre todo, la experiencia de aplicar estas metodologías de primera mano. De hecho, en la actualidad numerosas tesis y proyectos profesionales en ecología emplean exitosamente uno u otro de estos ciclos (figuras 1,2). En la práctica, en tres sitios fundadores de la Red Chilena de Estudios Socio-Ecológicos a Largo Plazo (LTSER) se pueden encontrar numerosos ejemplos de tesis y prácticas del Ciclo de Indagación. Tanto como otras metodologías de investigación, los dos ciclos pueden aplicarse en las ciencias sociales, además de las ciencias naturales. Por otro lado, la aparente sencillez de ambos ciclos (figuras 1,2 ) hace posible su uso por personas alejadas de la investigación académica (Arango et al. 2009, Feinsinger et al. 2010ab). ¿Eso es un defecto? Para ser rigurosa, ¿es necesario que la ciencia y su metodología sean redactadas en un lenguaje complejo y a menudo in- comprensible, entendido sólo por la elite de los científicos o epistemólogos más especializados? No necesariamente. Según R. H. MacArthur (1972), las únicas reglas del método científico son las observaciones honestas y la lógica exacta (...). Y al fin y al cabo, la ecuación más famosa de la física, $e=m c^{2}$, plasma un concepto revolucionario empleando un lenguaje sencillo y entendible por la mayoría.

\section{AGRADECIMIENTOS}

Agradezco mucho a J Armesto, J Astegiano, M Cock, R Rozzi, A Tálamo, C Trucco e I Ventosa Rodríguez su lectura crítica de borradores de este ensayo. Entre los miles de otros colegas y participantes en cursos cuyas ideas han contribuido al desarrollo del Ciclo de Indagación y el Ciclo de Indagación Aplicada cabe destacar a J Armesto, G Carreño, A Caselli, A Cormenzana y F Putz. RB Root y RH Whittaker sembraron las semillas del Ciclo de Indagación y el Ciclo de Indagación Aplicada, a la vez mostrándonos a nosotros sus alumnos que los dos elementos fundamentales de la metodología de investigación en Ecología son el conocimiento (y reconocimiento) de la historia natural, y el sentido común. Finalmente agradezco a L Marone y L Galetto su publicación del 2011, la cual ha sido un catalizador de este ensayo por ser una reinterpretación particular de un texto en inglés escrito en el 2001 y ya desbancado por el 2004, cuando salió la segunda edición en castellano.

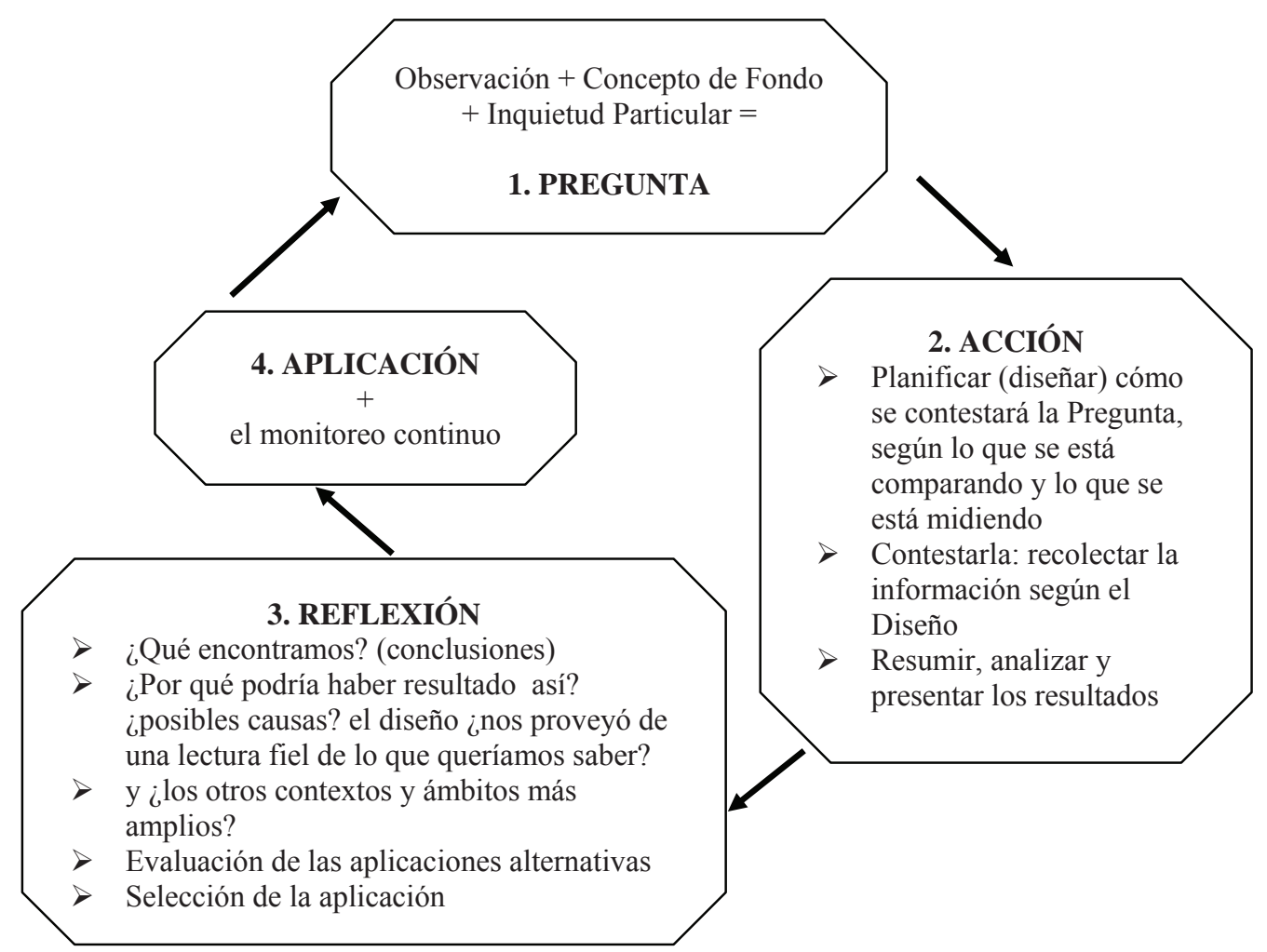

Figura 2. El Ciclo de Indagación Aplicada (modificado ligeramente de Feinsinger 2004 y Feinsinger et al. 2010a).

The Applied Inquiry Cycle (slightly modified from Feinsinger 2004, Feinsinger et al. 2010a). 


\section{REFERENCIAS}

Arango N, ME Chaves, P Feinsinger. 2009. Principios y práctica de la enseñanza de ecología en el patio de la escuela. Santiago, Chile. Instituto de Ecología y Biodiversidad - Fundación Senda Darwin. 135 p.

Connell JH. 1978. Diversity in tropical rain forests and coral reefs. Science 199: 1302-1310.

Farji-Brener AG. 2009. ¿Ecólogos o ególogos? Cuando las ideas someten a los datos. Ecología Austral 19: 167-172.

Feinsinger P, L Margutti, RD Oviedo. 1997. School yards and nature trails: ecology education outside the university. Trends in Ecology and Evolution 12: 115-120.

Feinsinger P. 2001. Designing field studies for biodiversity conservation. Washington DC, EEUU, Island Press. 212 p.

Feinsinger P. 2004. El diseño de estudios de campo para la conservación de la biodiversidad. Santa Cruz de la Sierra, Bolivia. Editorial FAN-Bolivia. 242 p.

Feinsinger, P, S Álvarez, G Carreño, E Rivera, RL Cuellar, A Noss, F Daza, M Figuera, L García, M Cañizares, A Alegre, A Roldán. 2010a. Local people, scientific inquiry, and the ecology and conservation of place in Latin America. In Billick I, MV Price eds. The ecology of place: contributions of place-base research to ecological and evolutionary understanding. Chicago, EE.UU. University of Chicago Press. p. 403-428.

Feinsinger P, C Pozzi, C Trucco, RL Cuellar, A Laina, M Cañizares, A Noss. 2010b. Investigación, conservación y los espacios protegidos de América Latina: una historia incompleta. Ecosistemas 19(2): 97-111.

Feinsinger P. 2012. Lo que es, lo que podría ser y el análisis e interpretación de los datos de un estudio de campo. Ecología en Bolivia 47: 1-6.

Feinsinger P. 2013. Metodologías de investigación en ecología aplicada y básica: ¿cuál estoy siguiendo, y por qué? Revista Chilena de Historia Natural 86: 385-402.

Feinsinger P, I Ventosa Rodríguez. 2014. Suplemento decenal al texto "El diseño de estudios de campo para la conservación de la biodiversidad”. Editorial FAN-Bolivia. 156 p.

Ford ED. 2000. Scientific method for ecological research. Cambridge, UK. Cambridge University Press. 588 p.

Fretwell SD. 1972. Populations in a seasonal environment. Princeton, EE.UU. Princeton University Press. 224 p.

Holling CS. 1978. Adaptive environmental assessment and management. Chichester, EE.UU. Wiley. 377 p.

Hurlbert SH. 2009. The ancient black art and transdisciplinary extent of pseudoreplication. Journal of Comparative Psychology 123: 434-443.

Lawton JH. 1999. Are there general laws in ecology? Oikos 84: 177-192.

Loehle C. 1987. Hypothesis testing in ecology: psychological aspects and the importance of theory. Quarterly Review of Biology 62: 397-409.

MacArthur RH. 1972. Geographical ecology: patterns in the distribution of species. New York, EE.UU. Harper \& Row. 288 p.

Marone L y L Galetto. 2011. El doble papel de las hipótesis en la investigación ecológica y su relación con el método hipotético-deductivo. Ecología Austral 21: 201-216.

Mead R. 1988. The design of experiments. Cambridge, UK. Cambridge University Press. 620 p.

Quinn GP, MJ Keough. 2002. Experimental design and data analysis for biologists. Cambridge, UK. Cambridge University Press. $537 \mathrm{p}$.

Quinn JF, AE Dunham. 1983. On hypothesis testing in ecology and evolution. American Naturalist 122: 602-617.

Ramage BS, D Sheil, HMW Salim, C Fletcher, NZA Mustafa, JC Luruthusamay, RD Harrison, E Butod, AD Dzulkiply, ABDR Kassim, MD Potts. 2013. Pseudoreplication in tropical forests and the resulting effects on biodiversity conservation. Conservation Biology 27: 364-372.

Shadish W, T Cook, D Campbell. 2002. Experimental and quasiexperimental designs for generalized causal inference. Boston MA, EE.UU. Houghton Mifflin. 623 p.

Underwood AJ. 1990. Experiments in ecology and management: their logics, functions and interpretations. Australian Journal of Ecology 15: 365-389. 
